\title{
Sub-Parts-per-Billion Determination of Nitro-Substituted Polynuclear Aromatic Hydrocarbons in Airborne Particulate Matter and Soil by Electron Capture-Tandem Mass Spectrometry
}

\author{
Marco Vincenti, Claudio Minero, and Ezio Pelizzetti \\ Dipartimento di Chimica Analitica, Università di Torino, Via Pietro Giuria, Torino, Italy \\ Marco Fontana and Roberta De Maria \\ Laboratorio di Sanitá Pubblica, Sezione Chimica, Via Leonardo da Vinci, Grugliasco (Torino), Italy
}

\begin{abstract}
A procedure for the determination of nitro-substituted polynuclear aromatic hydrocarbons (nitro-PAH) on crude air-particulate and soil extracts is introduced. Elimination of purification and fractionation procedures was made possible by the use of both a selective ionization method, such as electron-capture chemical ionization, and a specific fragmentation process, in an experiment of tandem mass spectrometry (gas chromatography-electron capture tandem mass spectrometry). Different mass spectrometric procedures were compared. The best performance was observed when the nitro-PAH molecular ions $[\mathrm{M}]^{-}$were mass-selected by the first analyzer under multiple reaction monitoring conditions and then fragmented to $\mathrm{NO}_{2}^{-}(\mathrm{m} / \mathrm{z} 46)$. Detection limits were on the order of hundreds of femtograms, as determined in extracts of real environmental samples. This corresponds approximately to 5-15 pg of nitro-PAH per cubic meter of air sampled. Calibration curves were linear over 3 orders of magnitude. Applications to contamination from motor vehicle combustion and the iron industry are briefly discussed. (c) 1996 American Society for Mass Spectrometry (J Am Soc Mass Spectrom 1996, 7, 1255-1265)
\end{abstract}

$\mathrm{N}$ itro-substituted polynuclear aromatic hydrocarbons (nitro-PAHs) are probably the most important class of mutagens identified as air pollutants in the last 15 years [1-8]. They originate from polynuclear aromatic hydrocarbons (PAHs) by at least three distinct processes, namely, (i) electrophilic nitration during combustion processes (particularly in diesel engines) [2, 5, 8-13], (ii) hydroxyl radical attack followed by reaction with $\mathrm{NO}_{x}$ and loss of a water molecule [8, 14-21], or (iii) reaction with $\mathrm{N}_{2} \mathrm{O}_{5}$ [22-24]. Both processes (ii) and (iii) take place during transport through the atmosphere by reaction of PAHs (generally adsorbed on particulate matter) with nitrogen oxides, but (ii) predominates during daylight hours, while (iii) is prevalent during the night. A further process has been repeatedly reported $[2,11,25-28]$ in which (iv) nitro-PAHs are artificially produced during particulate sample collection, although its relative importance recently has been cut down to size [28].

Address reprint requests and correspondence to Dr. Marco Vincenti, Dipartimento di Chimica Analitica, Università di Torino, Via Pietro Giuria, 510125 Torino, Italy.
Nitro-PAHs have been determined by means of bioassay-directed fractionation and chemical analysis [29] in particulate exhaust emission of motor vehicles $[5,9,10,13,30-42]$, airborne particulate matter [15-19, 27, 33, 34, 42-53], industrial emissions [49], carbon black, and toners $[3,54-56]$. All these matrices are extremely complex, since thousands of combustion products are generally present in the sample. Moreover, a number of parent PAH isomers and other closely related derivatives, which tend to coelute with nitro-PAHs under a variety of liquid and gas chromatographic conditions, are present at concentrations 1 or 2 order of magnitude higher than that of the nitrosubstituted compounds. Extensive workup procedures are required to isolate nitro-PAH from other classes of interfering compounds. The most widely used procedures for fractionation of particulate extracts include open column liquid chromatography either with silica gel or alumina [27, 32, 43-45], normal-phase high-performance liquid chromatography (HPLC) [13, 15-19, $28,33,34,41,46-49]$, and solid phase extraction [10, $37,57,58]$. From open column liquid chromatography four main fractions are generally obtained. In addition 
to nitro-PAHs, the moderately polar fraction contains also a number of oxy derivatives (aldehydes, ketones, quinones) [43] generally at a much higher concentration than nitro-PAHs, which may interfere with them, depending on the analytical technique utilized. Much more effective fractionation is achieved by normalphase HPLC, although nitro-PAHs are not collected in a single fraction, but rather they are distributed in several fractions, which may require separate analysis.

Even when nitro-PAHs have been partially isolated from the interfering substances and concentrated, the subsequent instrumental analysis has to be extremely sensitive and selective at once, since most nitro-PAHs prove to be mutagenic even at ultratrace concentrations. Selectivity and detection limits are closely linked together when extremely high chemical background is present in the sample, as is the case for real environmental samples, such as air particulate and soil. As a matter of fact, the detection limits often declared from experiments on nitro-PAH standard solutions did not hold when real environmental samples were considered. Among the techniques utilized to detect nitroPAHs, the most frequently used are gas chromatography combined with, respectively, a thermionic (TID) or nitrogen-phosphorus (NPD) selective detector [13, 15, $47,50,59]$, chemiluminescence-based thermal energy analyzer (TEA) [31-34] and electron capture detector (ECD) [44, 46, 57], HPLC with either fluorescence $[40-42,45]$, chemiluminescence $[36,51,56]$, or electrochemical $[37,42,43,58]$ detector, gas chromatography-mass spectrometry (GC-MS) in electron impact (EI) $[13,16-19,32,34,35,59]$, positive ion chemical ionization (PCI) [9], or negative ion chemical ionization (NCI) electron capture (EC) $[15,39,46,48,55,59]$.

A rather selective technique is electron capture chemical ionization mass spectrometry, as only the compounds with high electron affinity provide high response factors. Nitro-PAHs are among the organic compounds with the highest electron affinity [60]. The combination of polynuclear aromatic structure and strong electron-withdrawing character of the nitro group lowers the energy of the lowest unoccupied molecular orbital (LUMO) [60]. Moreover, electron capture-mass spectrometry (EC-MS) appears to yield the highest sensitivity for the most toxic among nitroPAHs, as their electron affinity and mutagenicity appear to be linked [8]. However, at the time when most applications of EC-MS to nitro-PAH determination were published, this technique was regarded as scarcely reproducible in terms of mass spectral features and response factors. Nowadays, EC-MS is a well-established technique that can be routinely used for accurate analytical determinations. Although GCEC-MS proved to provide adequate sensitivity and selectivity for the analysis of nitro-PAHs in most matrices, it still suffers from the interference of oxygenated PAHs, which have to be separated from nitro-PAHs by extensive sample cleanup and fractionation [31].
Tandem mass spectrometry (MS/MS) has occasionally been used in the analysis of nitro-PAHs, mostly without GC $[9,39]$. Schilhabel and Levsen [38] combined GC, EC, and MS/MS to achieve highly selective detection of nitro-PAHs, but full analytical characterization of the method was not attained. For example, the application for quantitative determinations has not been shown nor were detection limits and dynamic range obtained [38].

A survey of recent analytical chemistry literature reveals that interest in the research devoted to the detection of nitro-PAHs in environmental samples has progressively declined, after the explosion of the early 1980 s, despite the accumulation of a lot of evidence in the meantime that proved that nitro-PAHs are pollutants of enormous human health concern, large diffusion, and high environmental impact. The considerable progress of chemical intrumentation technology together with the substantial and quite generalized price cut on formerly expensive techniques, prompted us to reconsider this topic.

The rationale for choosing a GC-EC-MS/MS configuration is that it comprises four "separation" stages, namely, one chromatographic separation, a selective ionization method, and two mass spectrometric analyses. This leads to such a high selectivity of the instrumental technique that the preliminary sample fractionation is no longer required on air-particulate samples, thus avoiding both the need of time-consuming procedures and the risk of analyte loss during sample manipulation. We took up the instrument design proposed by Schilhabel and Levsen [38] for nitro-PAH analysis and expanded their objectives considerably. The removal of chemical interferences in the instrumental response is so efficient that the method competes with chemiluminescence detection [51] in terms of minimum detectable amount. Calibration curves proved to be linear over a 3 order-of-magnitude range. The performance of GC-EC-MS/MS for nitro-PAH determination was tested on several real environmental samples, including (i) air-particulate samples collected inside a foundry, (ii) one urban air-particulate sample, and (iii) several soil samples collected along a state highway.

\section{Experimental}

\section{Sample Preparation}

3-Nitrofluoranthene, 3-nitrophenanthrene, and 6nitrochrysene were purchased from Amchro Restek Europa GmbH (Sulzbach Taunus, Germany). The remaining nitro-PAH standards were obtained from Aldrich (Mannheim, Germany) and used without further purification. Standard solutions were prepared from the solid materials and subsequently diluted to the proper concentration.

Superficial soil samples were collected at a depth of $0-10 \mathrm{~cm}$ from the surface. Sampling sites were all at 
5-m distance from a state highway, but distributed along it at considerable distance from one another. The sample was homogenized and then a $20-\mathrm{g}$ aliquot was taken for the analysis. Soil sample extraction was carried out for $15 \mathrm{~h}$ in a Soxhlet device using a 1:1 (v/v) mixture of toluene and methanol. After Soxhlet extraction, part of the solvent was removed under mild conditions. The concentrated extract was passed through a silica-gel column $(5 \mathrm{~g})$ and eluted with 50 $\mathrm{mL}$ of toluene. The volume of resulting solution was reduced to $1 \mathrm{~mL}$ under a gentle stream of clean nitrogen before the analysis.

Collection of air particulates from the atmosphere inside a foundry was performed by a high-volume Staplex sampler, by using fiberglass filters, for total particulate sampling. The sampling was carried out at $1.5-\mathrm{m}$ height from the ground and went on for $200 \mathrm{~min}$ at a rate of approximately $150 \mathrm{~L} / \min$ (about $30 \mathrm{~m}^{3}$ of air sampled). The filters were subsequently extracted with $50 \mathrm{~mL}$ of toluene in an ultrasonic bath for $30 \mathrm{~min}$. The extract was concentrated to $1 \mathrm{~mL}$ under nitrogen and then analyzed, without further cleanup. The urban air particulate was sampled by using the same procedure as for the foundry particulate, except for the sampling time, which was $12 \mathrm{~h}$ (about $108 \mathrm{~m}^{3}$ of air sampled). The collection filter was subsequently extracted and analyzed. Three replicate analyses were carried out for each sample, on which standard deviations and calibration statistics were calculated.

\section{Extract Analysis}

All analyses were carried out by using a Finnigan-MAT $95 \mathrm{Q}$ (Bremen, Germany) hybrid tandem mass spectrometer, in which the magnetic, electrostatic, and quadrupole analyzers are mounted in sequence (BEQ geometry). An octapole collision cell is located between the electrostatic and the quadrupole sector to activate the parent ion dissociation in MS/MS experiments at low kinetic energy. A Varian 3400 (Palo Alto, CA) gas chromatograph is interfaced to the mass spectrometer.

Samples $(1 \mu \mathrm{L})$ were introduced into the split-splitless injector of the GC, kept at $300^{\circ} \mathrm{C}$, and worked in the splitless mode for $60 \mathrm{~s}$. The mixture was separated on a bonded-phase DB-5-MS capillary column (J \& W Scientific, Folsom, CA), 30-m-long, 0.25-mm internal diameter, and $0.25-\mu \mathrm{m}$ film thickness. The oven temperature was programmed as follows: isothermal at $90{ }^{\circ} \mathrm{C}$ for $3 \mathrm{~min}$; from 90 to $300^{\circ} \mathrm{C}$ at $12{ }^{\circ} \mathrm{C} / \mathrm{min}$; isothermal at $300{ }^{\circ} \mathrm{C}$ for $10 \mathrm{~min}$. The column end was introduced directly into the ion source of the mass spectrometer through a transfer line heated to $270^{\circ} \mathrm{C}$.

Ionization of the analytes eluted by the GC column was achieved by electron capture chemical ionization (EC). Isobutane was admitted into the ion source at a pressure of $50 \mathrm{~Pa}(0.5 \mathrm{mbar})$, while the electron emitting filament was kept at $200 \mathrm{~V}$ and the electron current at $0.2 \mathrm{~mA}$. The ion source temperature was maintained at $250^{\circ} \mathrm{C}$ to avoid condensation of the less volatile analytes. In the optimized procedure, negative ions generated in the ion source were accelerated to $5000 \mathrm{eV}$, analyzed by the magnetic sector, then driven through the electrostatic analyzer, and decelerated to $80 \mathrm{eV}$ by means of a series of cylindrical lenses. At this kinetic energy the ions underwent multiple collisions with $0.13 \mathrm{~Pa}$ of argon within the octapole collision cell, resulting in quite extensive fragmentation. Among the fragments generated by collision-induced dissociation (CID), only the ions of $m / z 46$ (basically, the $\mathrm{NO}_{2}^{-}$ ion) were mass-selected by the quadrupole filter and allowed to reach the detector. The first mass analyzer was programmed so as to perform cyclic mass-hopping among the molecular ions of target nitro-PAHs. This multiple reaction monitoring (MRM) experiment was set so that different time windows of the chromatographic run corresponded to different parent-ion selections, as follows: from 0 to $14.5 \mathrm{~min}, \mathrm{~m} / z 173$ only; from 14.5 to $19.25 \mathrm{~min}, \mathrm{~m} / z 211,218$, and 223; from $19.25 \mathrm{~min}$ to the end, $\mathrm{m} / z 247$ and 273 . With such programming, all nitro-PAHs could be detected in sequence as a function of their retention time. In a few experiments, the negative parent-ion spectra of $m / z 46$ were also recorded. This was achieved by continuously scanning the magnet from $\mathrm{m} / z 150$ to 300 in 0.8-s cycles, while keeping all the other parameters as in MRM experiments.

GC-EC-MS experiments were carried out under the same conditions adopted for MS/MS experiments, but the ions passed through a single mass analyzer and were detected at the first dynode-electron multiplier system, located after the electrostatic sector. The magnet was either continuously scanned or run under selected ion monitoring conditions, by using the same time windows as above. EI ionization was utilized in a few experiments at $70-\mathrm{eV}$ electron energy and $1.0-\mathrm{mA}$ electron current.

Neutral loss tandem mass spectra were obtained by scanning simultaneously the magnet and the quadrupole analyzer while keeping a constant difference (either 30 or $46 \mathrm{u}$ ) between the masses selected during the scan. The voltages of all the lenses that comprised the quadrupole system (deceleration and focusing lenses) were scanned together with the analyzer to ensure maximum ion transmission over the entire mass range. In these experiments, the laboratory collision energy and the collision gas pressure were set as in the MRM experiment described in the preceding text.

The collision gas pressure was optimized by measuring the fragment ion signal arising from CID at $0.05,0.13,0.2$, and $0.4 \mathrm{~Pa}$ of argon. Optimal collision energy was determined with the aid of breakdown graphs, built from consecutive experiments where only the voltage of the collision cell was varied. The intensity of the fragment ion signal was the result of both the fragmentation efficiency and the ion transmission. 


\section{Results and Discussion}

\section{Evaluation of the Method}

Collision-induced dissociation of nitro-PAH molecular ions generates various fragments, both in the positive and the negative ion mode. Positive molecular ions typically fragment by (i) loss of $\mathrm{NO}_{2}$, (ii) loss of $\mathrm{NO}$ followed by loss of $\mathrm{CO}$, and (iii) formation of $\mathrm{NO}^{+}$ ions. Quite similarly, negative molecular ions give rise to the following processes: (iv) loss of $\mathrm{NO}_{2}$, (v) loss of $\mathrm{NO}$, and (vi) formation of $\mathrm{NO}_{2}^{-}$ions. Any fragmentation process involving the nitro group could in theory be exploited in a tandem mass spectrometric experiment to achieve selective detection of nitro derivatives. In practice, the efficiency of the dissociation and its selectivity vary considerably from one process to another. The sensitivity and selectivity of processes (i), (ii), (iv), (v), and (vi) were compared by analyzing, under appropriate scarning of the two mass analyzers, both a soil sample extract (soil 51) containing several nitro-PAHs at trace level and a mixture of 11 nitro-PAH authentic standards at $100-\mu \mathrm{g} \mathrm{L}^{-1}$ concentration. As the various fragmentation processes are likely to have slightly different activation energies, some average collision energy and collision gas pressure were set (Experimental). Among the five experiments tested, the one yielding the best performance in term of specificity, sensitivity, and homogeneous response was, by far, process (vi). For all the other processes studied, several large interfering peaks were present in the chromatographic profile of the soil sample extract. This depended on the fact that fragmentation of highly concentrated extract components produced the same net mass loss as processes (i) and (iv) (neutral loss of $46 \mathrm{u}$.) and processes (ii) and (v) (neutral loss of $30 \mathrm{u}$ ). For example, consecutive losses of $\mathrm{H}_{2} \mathrm{O}$ and $\mathrm{CO}$ give rise to a net mass loss of $46 \mathrm{u}$ as for $\mathrm{NO}_{2}$, and a $\mathrm{HCHO}$ loss is equivalent, in terms of nominal mass, to a NO neutral loss.

The experiments performed on the $100-\mu \mathrm{g} \mathrm{L}^{-1}$ standard solution mixture showed that only experiment (i) produced comparable results as (vi) in terms of absolute signal, at least for mononitro-PAHs, but dinitronaphthalenes yielded almost no signal in experiment (i). Good sensitivity for dinitronaphthalenes was observed in both experiments (v) and (vi). On the other hand, (v) did not produce satisfactory results for mononitroPAHs. In practice, only the parent of $m / z 46$ negative ion scan (vi) proved adequate to detect selectively and with high signal-to-noise ratio all 11 nitro-PAHs tested, as the forthcoming figures demonstrate. This scan, either in the continuous mode or, more frequently, in the multiple reaction monitoring (MRM) mode, was the only one applied in the subsequent experiments on real environmental samples.

Several combinations of different collision gas pressures and collision energies were tested using the mixture of 11 standards. For most nitro-PAHs the best fragmentation efficiency was obtained for relatively low gas pressure $\left(1.3 \times 10^{-3} \mathrm{mbar}\right)$ and high laboratory collision energy $(80 \mathrm{eV})$. However, the fragmentation efficiency did not vary substantially over a quite large range of collisional energies, as the breakdown graph shown in Figure 1 demonstrates. Figure 1 reports the integrated signal (area) of single ion chromatographic peaks obtained by plotting the ion current relative to the fragmentation of each molecular ion $\left(\mathrm{M}^{+} \rightarrow \mathrm{NO}_{2}^{-}\right)$as a function of the laboratory collision energy. Although the fragmentation efficiency for 1nitronaphthalene and 9-nitroanthracene maximizes at collision energies lower than $80 \mathrm{eV}$, the signal loss suffered by operating at $80 \mathrm{eV}$ does not exceed $20 \%$. From Figure 1, it is also evident that the response factors for the various nitro-PAHs vary by up to almost 1 order of magnitude, due to several causes, including their different electron affinity, fragmentation efficiency, molar concentration (which is higher for light compounds at equal weight-to-weight concentration), and chromatographic injection and elution efficiencies. All these causes, with the exception of the first, tend to produce higher response factors for the nitro-PAHs with lower molecular weight. Breakdown curves are not reported for 1,8-dinitronaphthalene and 3-nitrofluoranthene, as their traces approximately overlap those of 2-nitrofluorene and 1-nitropyrene, respectively. Among dinitronaphthalenes, only the 1,5-isomer shows a response higher than mononitronaphthalenes, not the 1,3- and 1,8-isomers, possibly because their high electron affinity is partially compensated for by their relatively low fragmentation efficiency.

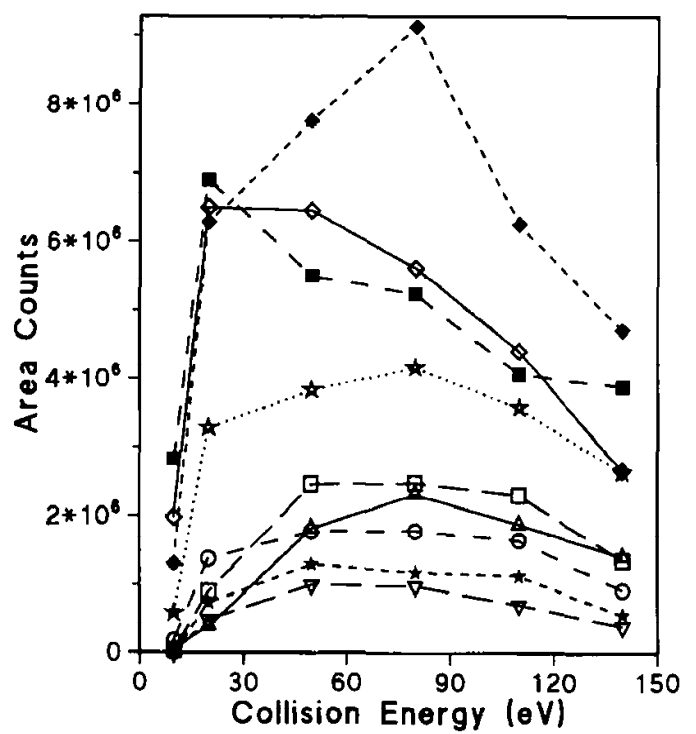

Figure 1. Breakdown curves representing the variation of the signal relative to the transition $[\mathrm{M}]^{-} \rightarrow\left[\mathrm{NO}_{2}\right]^{-}$as a function of laboratory collisional energy, for various nitro-PAHs. The response is provided by the integrated GC peak areas. $\bullet, 1,5-\mathrm{Di}$ nitronaphthalene; $\diamond$, 9-nitroanthracene; $\mathbf{\square}, 1$-nitronaphthalene; s, 2-nitronaphthalene; $\square, 3$-nitrophenanthrene; $\Delta, 2$ nitrofluorene; $O$, 1,3-dinitronaphthalene; $\star$, 1-nitropyrene; $\nabla$, 6-nitrochrysene. 
Figure 2 shows a comparison between MRM and continuous scanning methods of operating the first mass analyzer. Both chromatograms (a) and (b) were obtained by injecting $1 \mu \mathrm{L}$ of a $10-\mu \mathrm{g} \mathrm{L}^{-1}(\mathrm{ppb})$ standard solution. Chromatogram (a) is an elaboration of primary data, where the ion currents for only 6 ions (the molecular ions of 11 standards) were added together to produce a profile more closely comparable to that of Figure 2b. Even if all 11 standards in Figure 2a can be detected, the chromatographic trace is much more noisy than that obtained from the MRM experiment (Figure $2 b$ ). The spikes in the profile are occasionally as high as the signal obtained from 6-nitrochrysene, which is the analyte with the lowest response factor. Under such circumstances, the detection limit for most nitro-PAHs cannot be placed far beyond this concentration $\left(10 \mu \mathrm{g} \mathrm{L}^{-1}\right)$. In contrast, the chromatographic profile of Figure 2b appears to be extremely clean and free from spikes. The detection limits for the 11 standards (minimum detectable amounts from a pure standard mixture) have obviously to be found at much lower concentrations. Figure $2 \mathrm{c}$ presents the chromatogram obtained from a $500-\mathrm{ng} \mathrm{L}^{-1}(500 \mathrm{fg}$ injected) standard solution. The noise on the baseline is visible in this chromatogram, and precise evaluation of detection limits can be made. From repeated experiments at this and lower concentrations, cautious detection limits can be located in the range from 100 to 500 $\mathrm{fg}$, depending in the specific nitro-PAHs considered.

Calibration curves were obtained for 11 nitro-PAH standards (EC-MS/MS MRM experiments). They proved linear in the range from $500 \mathrm{fg}(500 \mathrm{ppt})$ to 200 pg. Calibration curves were obtained also for experiments carried out under EC-MS and EC-MS/MS (scan) conditions to compare EC-MS/MS MRM results with those obtainable using, respectively, a traditional procedure and the equivalent MS/MS scan suited for qualitative analysis. In Table 1 the calibration curves obtained from the different techniques are evaluated relative to one another by comparing their angular coefficient together with its standard deviation $\left(a \pm \sigma_{n}\right)$, the square of their correlation coefficient $\left(r^{2}\right)$, and the variance $\left(s^{2}\right)$ of single point deviations $\left(y-y_{\text {calculated }}\right)$ from the calibration line by using $(n-2)$ degrees of freedom. The correlation coefficient was excellent for MRM experiments and quite good also for the traditional EC-MS conditions, while $r^{2}$ turned out poor when continuous scanning of the magnetic mass analyzer was performed in the MS/MS configuration. Among the three mass spectrometry methods under comparison, the lowest $s^{2}$ values were obtained by the MRM experiments for 10 standards out of 11 , although the comparison with the values obtained by the EC-MS method does not show differences statistically significant at $95 \%$ confidence level ( $F$-test). In contrast, $s^{2}$ values yielded by the EC-MS/MS scan experiments are significantly higher ( $F$-test, $95 \%$ confidence level) than those obtained from EC-MS/MS MRM, at least for 9 standards out of 11 .
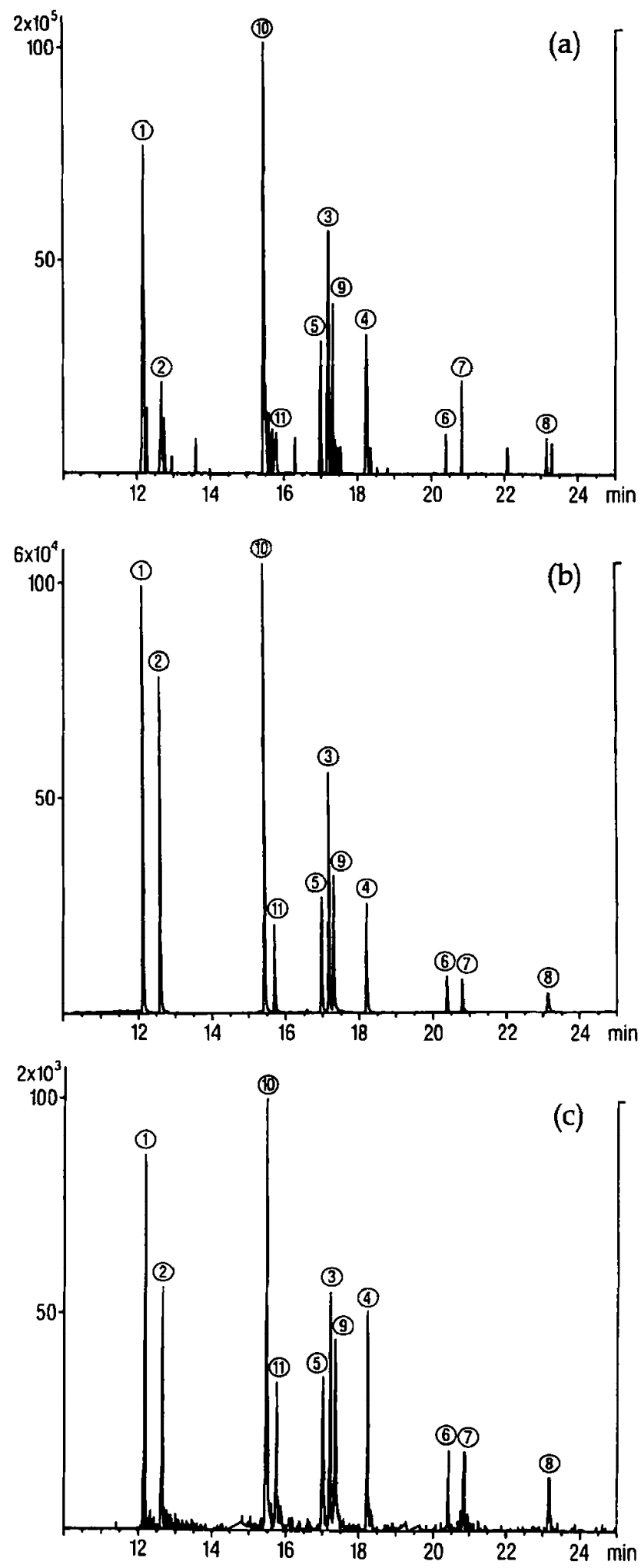

Figure 2. Gas chromatograms relative to the analysis of a mixture of 11 nitro-PAH standards by electron capture and tandem mass spectrometry. (a) 10-ppb solution under continuous scanning of the magnetic analyzer; (b) 10-ppb solution under MRM mode; (c) 500-ppt solution under MRM mode. Nitro-PAHs are indicated as follows: (1), 1-nitronaphthalene; (2), 2-nitronaphthalene; (3), 9-nitroanthracene; (4), 3-nitrophenanthrene; (5), 2-nitrofluorene; (6), 3-nitrofluoranthene; (7), 1-nitropyrene; (8), 6-nitrochrysene; (9), 1,8-dinitronaphthalene; (10), 1,5-dinitronaphthalene; (11), 1,3-dinitronaphthalene. 
Table 1. Calibration curves for 11 nitro-PAHs obtained from least squares linear fit of bilogarithmic plots (GC peak area versus concentration) ${ }^{a}$

\begin{tabular}{|c|c|c|c|c|c|c|c|c|c|}
\hline & \multicolumn{3}{|c|}{ EC-MS/MS MRM $(0.5-200 \mathrm{pg})$} & \multicolumn{3}{|c|}{ EC-MS/MS scen $(10-1000 \mathrm{pg})$} & \multicolumn{3}{|c|}{ EC-MS $(5-1000 \mathrm{pg})$} \\
\hline & $a \pm \sigma_{a}$ & $r^{2}$ & $s^{2}$ & $a \pm \sigma_{a}$ & $r^{2}$ & $s^{2}$ & $\theta \pm \sigma_{s}$ & $r^{2}$ & $s^{2}$ \\
\hline Nitr & $1.017 \pm 0.018$ & 0.9980 & 000 & $1 \pm 0.093$ & 29 & 2225 & $.040 \pm 0.035$ & 45 & .00456 \\
\hline 2-Nitronaphthalene & $1.039 \pm 0.027$ & 0.9960 & 0.00469 & $1.247 \pm 0.081$ & 9835 & .01693 & $121 \pm 0.046$ & 0.9918 & .00792 \\
\hline Nitroanthracene & $0.996 \pm 0.027$ & 0.9977 & 0.00484 & $1.126 \pm 0.049$ & 0.9924 & 0.00629 & $1.144 \pm 0.054$ & 0.9890 & .01108 \\
\hline Nit & $1.064 \pm$ & 0.9981 & 0.00231 & $1.141 \pm$ & 0.9660 & 0.02970 & $1.199 \pm 0$ & 0.9965 & .00382 \\
\hline Nit & $1.029=$ & 0.9976 & 0.00538 & 1.109 & 0.9599 & 324 & $1.206 \pm$ & 0.9 & .00796 \\
\hline 1 - Nit & 1.123 & 0.9979 & 0.0 & 1.0 & 83 & & $1.297 \pm$ & 0. & 24 \\
\hline 3-Nitr & $1.063=$ & 0.9974 & 0.00319 & $1.164 \pm$ & 0.9796 & & $1.326 \pm$ & & 15 \\
\hline Nit & 43 & .9913 & 83 & $175+$ & .9415 & 546 & $1.267 \pm C$ & 0.9744 & 0.03 \\
\hline $5-\mathrm{C}$ & 1.05 & 0.9985 & 84 & 1.153 & 9777 & 963 & $1.148 \pm$ & 0.9 & 0.00267 \\
\hline $1,8-\mathrm{D}$ & 1.0 & 0.9962 & & & 81 & & $41 \pm c$ & 0.5 & .00435 \\
\hline 1,3-Dinitr & $1.067 \pm$ & 0.9970 & 0. & $1.289 \pm$ & 20 & & $1.298 \pm c$ & 68 & 0405 \\
\hline $\begin{array}{l}\text { erage for } \\
\text { nitro-PAHs }\end{array}$ & $1.058 \pm 0.025$ & 0.9969 & 0.00430 & $1.159 \pm 0.099$ & 0.9693 & 0.02680 & $1.208 \pm 0.042$ & 0.9931 & 0.00780 \\
\hline
\end{tabular}

The calibration range for each different instrument configuration is indicated. For each nitro-PAH, the curve fit is represented by the angular coefficient $(a)$. its standard deviation $\left(\sigma_{s}\right)$ the square of the correlation coefficient $\left(r^{2}\right)$, and the variance of the vertical deviations from the calibration curve $\left(s^{2}\right)$

In a logarithmic plot (Figure 3) the angular coefficients (a) of the calibration curves obtained from the MRM procedure were not far from 1 (average 1.058), as was expected. However, these angular coefficients were determined with relatively high precision (low $\sigma_{a}$ values), making the difference $(1-a)$ statistically significant at the $95 \%$ confidence level for 6 standards out of 11 ( $t$-test). Angular coefficients largely exceeding unity were obtained from both EC-MS and EC-MS/MS scan experiments, corresponding to a too-rapid decline of the analytical signal with the decrease of nitro-PAH concentration. These $(a)$ values, averaged for the 11 standards (1.208 and 1.159, respectively, see Table 1), exhibit large differences also from the average angular coefficient determined under the MRM procedure (1.058). These differences are statistically significant at

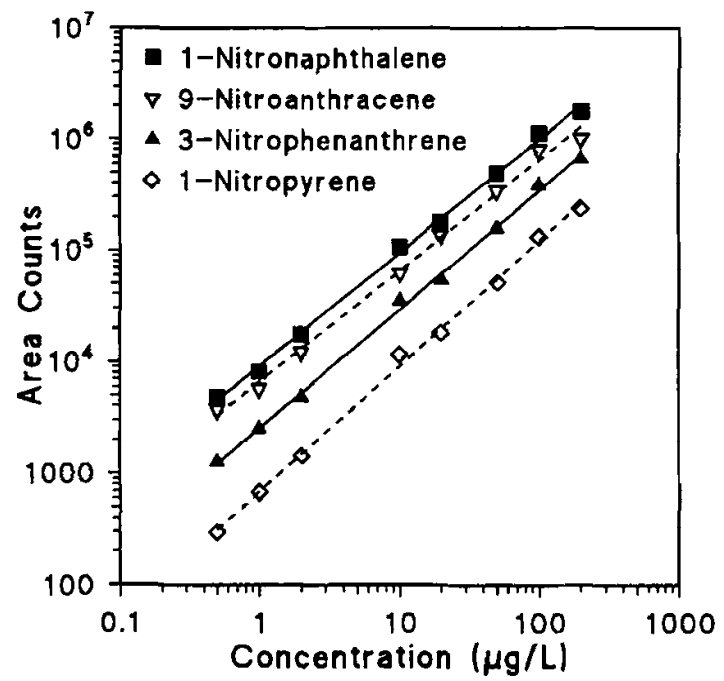

Figure 3. Bilogarithmic calibration graphs of GC peak areas versus concentration for four nitro-PAH standards. Counts are relative to $\left[\mathrm{NO}_{2}\right]^{-}$ions produced by collision induced dissociation of negatively charged molecular ions. the $95 \%$ confidence level ( $t$-test), demonstrating the nonequivalence of the mass spectrometry procedures under comparison.

System performance was checked daily by injecting a 500-ppt standard mixture. The calibration curves proved stable during a 3-week period. Apparently, calibration curves remained valid until the chromatographic performance was maintained.

\section{Soil Samples}

One soil sample (soil $\mathrm{T} 66$ ) was split and one-half of it was spiked with the nitro-PAH standard mixture so as to yield an extract supplemented by $2 \mu \mathrm{g} \mathrm{L}^{-1}$ of each authentic standard (approximately $100 \mathrm{ppt}$ in the soil). This allowed us to check the recovery and to confirm the detection limits on a real sample, at least for the analytes not already present in it. Figure 4 shows the chromatograms obtained from the extracts of both the unspiked (a) and the spiked (b) samples, while the quantitative results are reported in Table 2 (column $T$ 66 and $\mathrm{T} 66^{*}$ ). Both chromatograms display a quite neat baseline from which the peaks relative to nitroPAHs stand out. Even if there are more peaks in Figure $4 \mathrm{~b}$ than in Figure $4 \mathrm{a}$ and these peaks exhibit higher intensity, nonetheless the main features of the chromatograms are the same. Quantitative results (Table 2) confirm that most nitro-PAHs were already present in the unspiked sample, but their abundance was enhanced by the spiking. For example, the two nitronaphthalenes and 1-nitropyrene increased from 3 to $5 \mathrm{ppb}$, as expected, while several other analytes passed from hundreds of parts per trillion to $2 \mathrm{ppb}$. Dinitro-PAHs (not reported in Table 2) were absent in the unspiked sample, but were detected at 2-ppb concentration in the spiked extract.

The signal-to-noise ratio in the two chromatograms of Figure 4 is approximately the same as in the chromatogram from the standard mixture, which proves 
Table 2. Quantitative determination of nitro-PAHs in soil extracts ${ }^{a}$

\begin{tabular}{lccccccccc}
\hline & \multicolumn{1}{c}{ Soil samples } \\
\cline { 2 - 9 } Samples & T66 & T 66* & T0 & T7 & A 4 & 15 & 16 & 51 & 54 \\
\hline \hline 1-Nitronaphthalene (1) & $3.0 \pm 0.3$ & $5 \pm 0.6$ & $1.7 \pm 0.3$ & $1.9 \pm 0.2$ & $2.0 \pm 0.2$ & $1.2 \pm 0.1$ & $1.7 \pm 0.3$ & $1.7 \pm 0.1$ & $1.1 \pm 0.1$ \\
2-Nitronaphthalene (2) & $3.1 \pm 0.4$ & $5 \pm 0.5$ & $1.7 \pm 0.2$ & $2.2 \pm 0.2$ & $2.4 \pm 0.1$ & $1.7 \pm 0.2$ & $2.1 \pm 0.3$ & $2.2 \pm 0.2$ & $1.5 \pm 0.1$ \\
9-Nitroanthracene (3) & $0.6 \pm 0.1$ & $2.2 \pm 0.3$ & $<0.5$ & $<0.5$ & $<0.5$ & $<0.5$ & $<0.5$ & $<0.5$ & $<0.5$ \\
3-Nitrophenanthrene (4) & $0.7 \pm 0.1$ & $2.6 \pm 0.2$ & $0.7 \pm 0.1$ & $0.8 \pm 0.1$ & $0.5 \pm 0.1$ & $0.5 \pm 0.1$ & $0.7 \pm 0.1$ & $<0.5$ & $<0.5$ \\
2-Nitrofluorene (5) & $<0.5$ & $2.2 \pm 0.4$ & n.d. & n.d. & n.d. & n.d. & n.d. & n.d. & n.d. \\
3-Nitrofluoranthene (6) & $3.0 \pm 0.4$ & $4 \pm 0.7$ & $8 \pm 0.9$ & $8 \pm 1.1$ & $5 \pm 0.6$ & $8 \pm 0.6$ & $9 \pm 1.2$ & $7 \pm 0.5$ & $3.1 \pm 0.4$ \\
1-Nitropyrene (7) & $3.2 \pm 0.4$ & $5 \pm 0.7$ & $6 \pm 0.7$ & $6 \pm 0.8$ & $\mathbf{8} \pm 0.7$ & $9 \pm 0.9$ & $10 \pm 1.0$ & $10 \pm 0.9$ & $5 \pm 0.6$ \\
6-Nitrochrysene (8) & $0.6 \pm 0.2$ & $2.0 \pm 0.3$ & n.d. & n.d. & n.d. & $<0.5$ & n.d. & $0.5 \pm 0.2$ & n.d. \\
\hline
\end{tabular}

${ }^{a}$ The results are expressed as micrograms per liter (ppb) in the extract. The concentration factor from soil to extract is $20: 1$ ( $w / w$ ). Detectable analytes whose concentration is below the range of the calibration curve are indicated as < 0.5 . Undetected analytes are indicated as n.d.
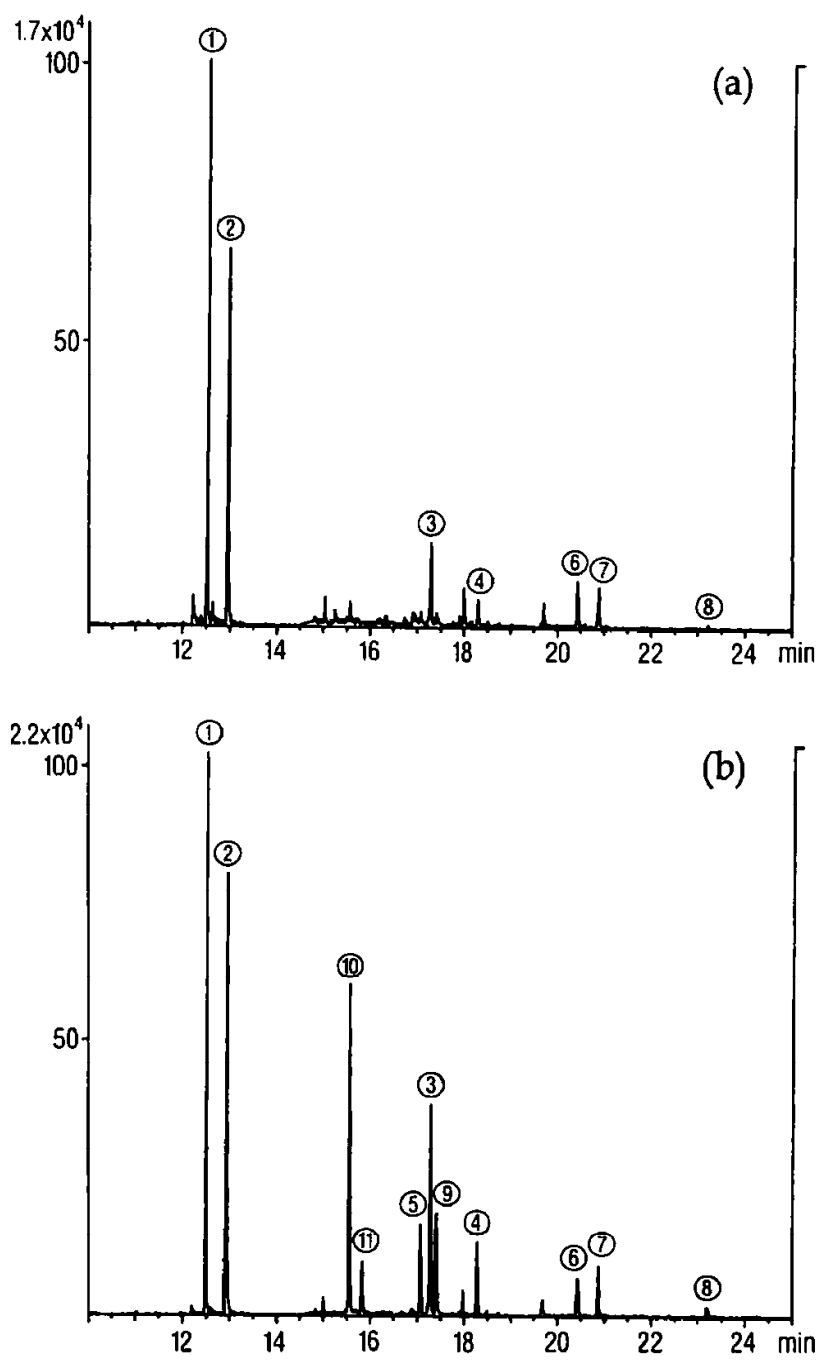

Figure 4. (a) Gas chromatogram of soil T 66 sample extract, as analyzed by the optimized EC-MS/MS MRM procedure. (b) Gas chromatogram of soil T 66 sample extract, spiked with 11 nitroPAH at 2-ppb level each. Nitro-PAHs are indicated as in Figure 2. that the MS/MS procedure adopted is very effective in removing the chemical noise produced by the complex matrices. Only in the range of retention times between 14 and $18 \mathrm{~min}$ is the baseline slightly more noisy than in the profile of the standard solution, but the nitroPAHs whose retention time is encompassed in this time range exhibit good response factors. It can be concluded that the assumption of a $500-\mathrm{ng} \mathrm{L}^{-1}$ (ppt) detection limit is reliable also for all 11 nitro-PAHs in the real environmental samples considered in the present study. Even if the analytes could occasionally be quantified at concentrations below these detection limits, they were indicated as $<0.5 \mu \mathrm{g} \mathrm{L}^{-1}$ in Table 2, provided that they were attributed with certainty.

The chromatograms reported in Figure 4 show three small peaks, among the others, with retention times of approximately 15.0, 18.0, and $19.7 \mathrm{~min}$, that do not correspond to any of the 11 standards. Even if their unequivocal identification required comparison with the corresponding authentic standards, they can tentatively be attributed to positional isomers of nitrofluorene, nitrophenanthrene, and nitrochrysene. If this is the case, they can be accounted for as analytes, not as interferences.

Clear evidence of the complexity of the matrix and the high specificity obtained by using the MS/MS procedure is provided by Figure 5, where the gas chromatograms obtained by applying different instrumental techniques to the analysis of the same soil extract (soil 51) are compared. The GC/MS analysis executed in EI mode with continuous scanning of the mass analyzer produced a GC trace (Figure 5a) showing the presence of hundreds (possibly thousands) of unresolved peaks, none of which corresponds to nitro$\mathrm{PAH}$. It is worth noting that the solution injected was not a crude extract; it had been roughly purified by elution on a silica-gel column. Nonetheless, the main components of the matrix produced such an intense signal that it prevented the detection of nitro-PAHs, which were present at trace level. The same result 

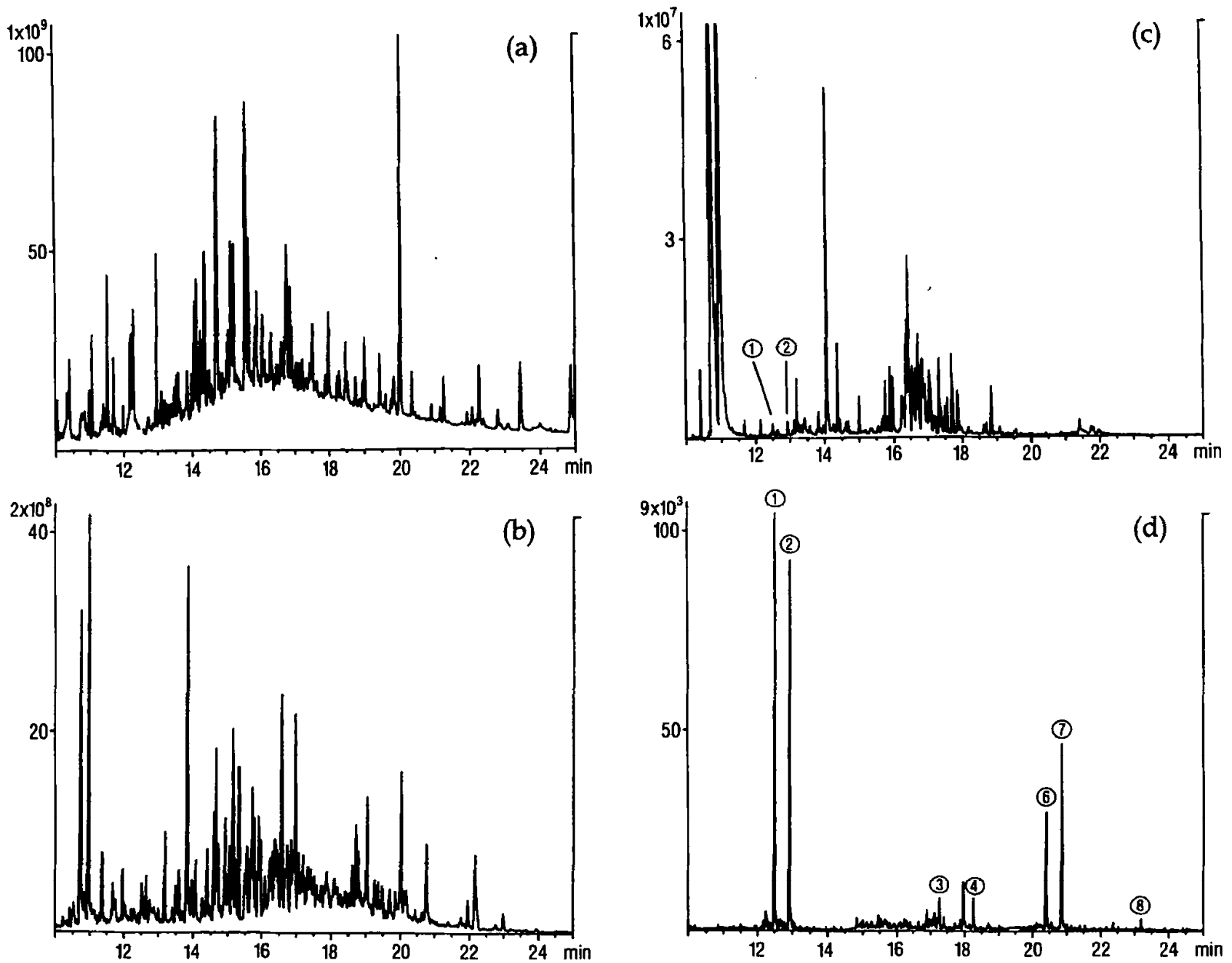

Figure 5. Gas chromatograms of soil 51 sample extract under various experimental conditions. (a) EI-MS scan; (b) EC-MS scan; (c) EC-MS SIM; (d) EC-MS/MS MRM. Nitro-PAHs are indicated as in Figure 2.

occurred when the sample was analyzed by EC-MS under continuous scanning of the mass analyzer (Figure $5 \mathrm{~b}$ ), although the baseline is smoother and the peaks come forth from it more neatly. A clear improvement was obtained by combining EC with selected ion monitoring (SIM), where the mass analyzer was allowed to monitor only the six molecular ions of interest. Despite the evident simplification of the GC trace (Figure 5c), still all the most intense peaks correspond to interfering substances, while only two minor peaks can be attributed to nitro-PAHs namely, 1- and 2nitronaphthalenes, indicated by an arrow in Figure $5 c$. In sharp contrast to the preceding situations, the execution of the EC-MS/MS (MRM) experiment removed completely the chemical noise from the GC trace (Figure 5d), leaving the nitro-PAH signals free from any interference. Under such circumstances, 1- and 2nitronaphthalene produced very intense peaks corresponding to a $2-\mu \mathrm{g} \mathrm{L}^{-1}$ concentration, as did also 3-nitrofluoranthene and 1-nitropyrene, present at 7and $10-\mu \mathrm{g} \mathrm{L}^{-1}$ levels, respectively. Even 9- nitroanthracene, 3-nitrophenanthrene, and 6-nitrochrysene, present in the extract at 500-ppt concentration or below (see Table 2) could be easily detected and quantified in the GC trace (Figure $5 \mathrm{~d}$ ). The comparison between Figure $5 \mathrm{c}$ and $\mathrm{d}$ clearly demonstrates the advantage of adding a second mass-separation gate to the already selective GC-EC-MS (SIM) analysis.

A survey of Table 2 reveals that the eight analyzed samples possess a rather uniform composition, at least with respect to the nitro-PAH content. As a matter of fact, the samples were collected at about the same distance from the road $(5 \mathrm{~m})$, though at large distance from one another. This fact, together with the large abundance of 1-nitropyrene and 3-nitrofluoranthene, suggests that soil contamination was mainly due to the direct deposition of particulate matter from exhaust emission of motor vehicles, not to (photoassisted) processes at the gas-solid interface. However, the environmental implications of these analyses are out of the scope of the present study, which focuses on the implementation of adequate analytical procedures for ni- 


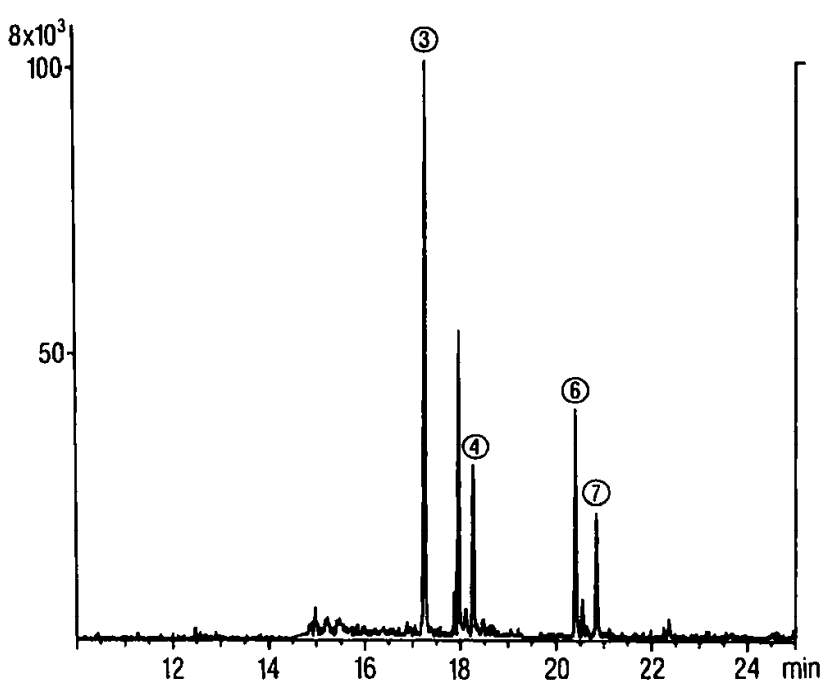

Figure 6. Gas chromatogram of air-particulate F 35 sample extract, obtained from EC-MS/MS MRM conditions. Nitro-PAHs are indicated as in Figure 2.

tro-PAH detection. With respect to this point, the developed procedure appears to achieve high sensitivity and high selectivity at the same time, and it also provides the molecular weight information, which is useful for structural confirmation. The removal of the chemical interferences arising from the soil matrix allows detection limits below $500 \mathrm{ng} \mathrm{L}^{-1}$ to be obtained in the extracts, corresponding to approximately $25 \mathrm{ppt}$ in the starting soil samples. This means that each nitro-PAH can be determined when its concentration exceeds $25 \mathrm{pg} \mathrm{g}^{-1}$ of soil.

\section{Air-Particulate Samples}

Ten air-particulate samples were collected within a single foundry plant, but at different positions of the steel and pig iron processing. The collection glass filters were extracted and the extracts were concentrated and analyzed directly, as cleanup procedures proved unnecessary. Figure 6 shows the gas chromatogram obtained from sample F35 employing the usual ECMS/MS (MRM) settlement. Five major peaks are present, four of which are identified as, respectively, 9-nitroanthracene, 3-nitrophenanthrene, 3-nitrofluoranthene, and 1-nitropyrene. The fifth peak is eluted at $18.0 \mathrm{~min}$ and exhibits the same molecular weight as nitroanthracene and nitrophenanthrene. From literature data [20] (reactivity and chromatograms) the fifth peak can be tentatively attributed to 9-nitrophenanthrene. Also five other minor peaks are likely to be positional isomers of the nitro-PAH standards examined in the present study: three are possibly further isomers of nitrophenanthrene or nitroanthracene, one (r.t. $20^{\prime} 33^{\prime \prime}$ ) can be attributed to 8-nitrofluoranthene [9, 53] and the fifth has the molecular weight of nitrochrysene. No other interfering peaks are present and the background noise is very limited, as already observed for soil sample extracts.

Table 3 reports the quantitative results from the particulate samples collected within the foundry. Two of the samples were split and spiked with the nitroPAH standard mixture, yielding extracts fortified with $2 \mu \mathrm{g} \mathrm{L}^{-1}$ of each analyte. While in no case did the analytical procedure fail to detect the added analytes, the quantification of this spiking turned out to be inaccurate only for the analytes whose concentration was already high in the unspiked sample (i.e., 9nitroanthracene and 3-nitrofluoranthene in F 46). For all the other analytes, good recovery and quantification was observed. The results on spiked samples prove that the accuracy of the method is satisfactory and that for none of the 11 standards is signal suppression from possibly coeluting substances observed. This, however, does not exclude that specific matrix components may capture substantial fractions of thermal electrons at retention times where none of the standards is eluted.

The nitro-PAH concentrations at the various working posts exhibited a wide variability. For example, 9-nitroanthracene ranged from 1 (F 69) to $100 \mathrm{ppb}$ (F 46) and 1-nitropyrene from 0.8 (F 303) to $11 \mathrm{ppb}$ (F 36). In general, some working posts could be classified as safe with regard to nitro-PAH pollution (F 69, F 69b, F 288), since their total concentration was quite low, while in other posts (F 46, F 36) such heavy air contamination was found to raise serious concern about the

Table 3. Quantitative determination of nitro-PAHs in air-particulate extracts ${ }^{\mathbf{a}}$

\begin{tabular}{|c|c|c|c|c|c|c|c|c|c|c|c|c|c|}
\hline \multirow[b]{2}{*}{ Samples } & \multicolumn{13}{|c|}{ Air-particulate sample } \\
\hline & F 36 & $F 36$ & $F 36^{*}$ & $F 46$ & $F 46^{*}$ & F 35 & $F 45$ & F 69 & $F 69 b$ & F 288 & $F 301$ & F 302 & F 303 \\
\hline 1-Nitronaphthalene (1) & $<0.5$ & $<0.5$ & $2.2 \pm 0.3$ & $1.6 \pm 0.3$ & $3.5 \pm 0.4$ & $<0.5$ & $<0.5$ & $<0.5$ & $<0.5$ & $<0.5$ & $0.5 \pm 0.1$ & $<0.5$ & $<0.5$ \\
\hline 2-Nitronaphthalene (2) & $<0.5$ & $<0.5$ & $2.6 \pm 0.4$ & $0.7 \pm 0.1$ & $3.2 \pm 0.4$ & $<0.58$ & $<0.5$ & $<0.5$ & $<0.5$ & $<0.5$ & $0.8 \pm 0.2$ & $<0.5$ & $<0.5$ \\
\hline 9-Nitroanthracene (3) & $5 \pm 0.7$ & $5 \pm 0.7$ & $7 \pm 0.8$ & $110 \pm 18$ & $100 \pm 16$ & $2.7 \pm 0.4$ & $11 \pm 2$ & $1.6 \pm 0.3$ & $2.6 \pm 0.3$ & $1.8 \pm 0.2$ & $3.2 \pm 0.4$ & $11 \pm 1.2$ & $6 \pm 0.7$ \\
\hline 3-Nitrophenanthrene (4) & $3.1 \pm 0.4$ & $3.1 \pm 0.4$ & $6 \pm 0.7$ & $7 \pm 0.9$ & $10 \pm 1.3$ & $1.9 \pm 0.3$ & $1.0 \pm 0.2$ & n.d. & $0.5 \pm 0.1$ & $0.6 \pm 0.1$ & $<0.5$ & $0.8 \pm 0.2$ & $0.5 \pm 0.1$ \\
\hline 2-Nitrofluorene (5) & n.d. & n.d. & $3.0 \pm 0.5$ & n.d. & $3.1 \pm 0.5$ & n.d. & n.d. & n.d. & n.d. & n.d. & n.d. & n.d. & n.d. \\
\hline 3-Nitrofluoranthene (6) & $13 \pm 2$ & $13 \pm 2$ & $17 \pm 3$ & $25 \pm 5$ & $20 \pm 4$ & $8 \pm 1.5$ & $7 \pm 1.2$ & $1.2 \pm 0.2$ & $1.3 \pm 0.2$ & $4 \pm 0.7$ & $2.6 \pm 0.4$ & $7 \pm 1.0$ & $10 \pm 2$ \\
\hline 1-Nitropyrene (7) & $11 \pm 2$ & $11 \pm 2$ & $16 \pm 3$ & $5 \pm 0.8$ & $8 \pm 1.5$ & $4 \pm 0.6$ & $2.5 \pm 0.4$ & $2.8 \pm 0.5$ & $1.5 \pm 0.3$ & $2.5 \pm 0.3$ & $1.7 \pm 0.3$ & $1.9 \pm 0.2$ & $0.8 \pm 0.1$ \\
\hline 6-Nitrochrysene (B) & $0.8 \pm 2$ & $0.8 \pm 0.2$ & $2.9 \pm 0.4$ & $0.9 \pm 0.2$ & $3.0 \pm 0.4$ & n.d. & n.d. & n.d. & n.d. & n.d. & $<0.5$ & $0.5 \pm 0.2$ & n.d. \\
\hline
\end{tabular}

- The results are expressed as micrograms per liter $(\mathrm{ppb})$ in the extract. The concentration factor from air sampled to extract is $30: 1\left(\mathrm{~m}^{3} / \mathrm{g}\right)$. Detectable analytes whose concentration is below the range of the calibration curve are indicated as $<0.5$. Undetected analytes are indicated as $n . d$. 
working conditions. For the specific foundry considered in our study, these samples were collected at sites close, respectively, to the casting and the shakeout stages of pig iron processing.

As for soil samples, $0.5 \mu \mathrm{g} \mathrm{L}^{-1}$ was assumed as the detection limit for nitro-PAHs in the extract. This concentration corresponds to $500 \mathrm{fg}$ injected $(1 \mu \mathrm{L})$ and to $15 \mathrm{pg} / \mathrm{m}^{3}$ of air sampled, much below the concentrations usually allowed for most air contaminants (i.e., PAHs) by the regulatory laws of industrialized countries.

A single urban air-particulate sample was collected during summertime at a road intersection in Rivoli (Turin metropolitan area) to verify the applicability of our procedure to the problem of urban air quality control. This sample was collected and treated the same as the foundry samples except for the larger air volume sampled $\left(108 \mathrm{~m}^{3}\right.$ instead of $\left.30 \mathrm{~m}^{3}\right)$. The chromatographic profile obtained from the corresponding extract is shown in Figure 7. A few peaks appear in the chromatogram, six of which coincide with nitro-PAH authentic standards: the two nitronaphthalenes were quantified as approximately $0.5 \mathrm{ppb}\left(4.5 \mathrm{pg} / \mathrm{m}^{3}\right) ; 9-$ nitroanthracene and 3-nitrophenanthrene as $2 \mathrm{ppb}$ $\left(18 \mathrm{pg} / \mathrm{m}^{3}\right)$; 1-nitropyrene as $10 \mathrm{ppb}\left(90 \mathrm{pg} / \mathrm{m}^{3}\right) ; 3$ nitrofluoranthene as $60 \mathrm{ppb}\left(550 \mathrm{pg} / \mathrm{m}^{3}\right)$. Thus, the heavy nitro-PAHs are prevalent in the particulate, as is expected for the condensate phase during the warm season [19]. The specific nitrofluoranthene and nitropyrene isomers found in the air particulate clearly indicate their origin in the electrophilic nitration of the corresponding PAHs, during motor-vehicle combustion processes [20]. Extensive work on the presence and formation mechanisms of nitro-PAHs in the urban air particulate and atmosphere of our metropolitan area is in progress.

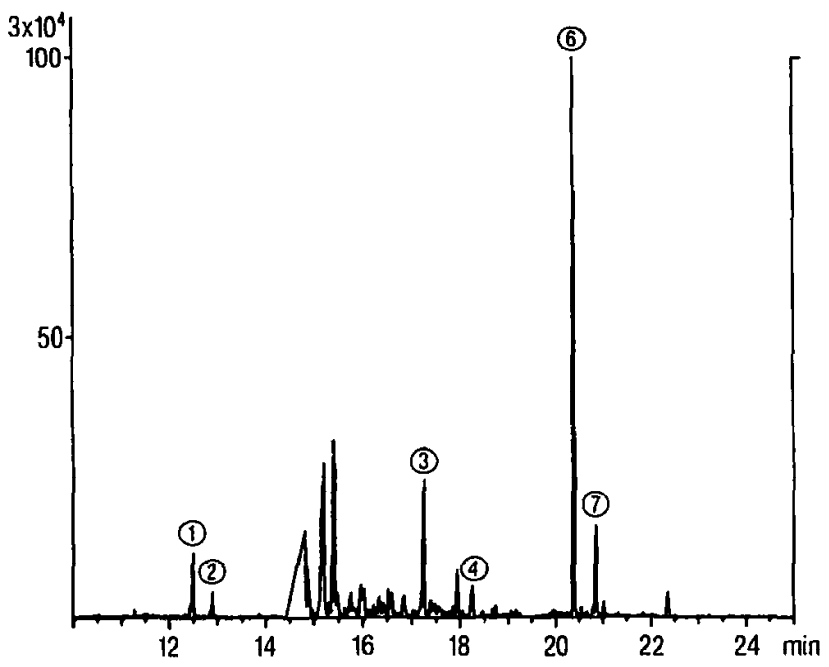

Figure 7. Gas chromatogram obtained from an urban airborne particulate sample extract under EC-MS/MS MRM conditions. Nitro-PAHs are indicated as in Figure 2.

\section{Acknowledgments}

The financial support of Provincia di Torino, Regione Piemonte, M.U.R.S.T., and C.N.R. is gratefully acknowledged.

\section{References}

1. Ames, B. N.; McCann, J.; Yamasaki, E. Mutat. Res. 1975, 31, 347-364.

2. Pitts, J. N., Jr.; Cauwenberghe, K. A.; Grosjean, D.; Schmid, J. T.; Fitz, D. R.; Belser, W. L., Jr.; Knudson, G. B.; Hynds, P. M. Science 1978, 202, 515-519.

3. Rosenkranz, H. S.; McCoy, E. C.; Sanders, D. R.; Butler, M.; Kiriazides, D. K.; Mermelstein, R. Science 1980, 209, 1039-1043.

4. Pederson, T. C.; Siak, J.-S. J. Appl. Toxicol. 1981, 1, 54-166.

5. Schuetzle, D.; Lee, F. S.-C.; Prater, T. J.; Tejada, S. B. Int. J. Environ. Anal. Chem. 1981, 9, 93-144.

6. Salmeen, I. T.; Pero, A. M.; Zator, R.; Schuetzle, D.; Riley, T. L. Environ. Sci. Technol. 1984, 18, 375-382.

7. Rosenkranz, H. S.; Mermelstein, R. In Nitrated Polycyclic Aromatic Hydrocarbons; White, C. M., Ed.; Huetig: Heidelberg, Germany, 1985; pp 267-298.

8. Lewtas, J.; Nishioka, M. G. In Nitronrenes; Howard, P. C., Ed.; Plenum Press: New York, 1990; pp 61-72.

9. Schuetzle, D.; Riley, T. L.; Prater, T. J.; Harvey, T. M.; Hunt, D. F. Anal. Chem. 1982, 54, 265-271.

10. Xu, X. B.; Nachtman, J. P.; Jin, Z. L.; Wei, E. T.; Rappaport, S. M.; Burlingame, A. L. Anal. Chim. Acta 1982, 136, 163-174.

11. Pitts, J. N., Ir.; Lokensgard, D. M.; Harger, W.; Fisher, T. S.; Mejia, V.; Schuler, J. J.; Scorziell, G. M.; Katzenstein, Y. A. Mutat. Res. 1982, 103, 241-249.

12. Newton, D. L.; Erickson, M. D.; Tomer, K. B.; Pellizzari, E. D.; Gentry, P.; Zweldinger, R. B. Environ. Sci. Technol. 1982, 16, 206-213.

13. Paputa-Peck, M. C.; Marano, R. S.; Schuetzle, D.; Riley, T. L.; Hampton, C. V.; Prater, T. J.; Skewes, L. M.; Jensen, T. E.; Ruehle, P. H.; Bosch, L. C.; Duncan, W. P. Anal. Chem. 1983, 55, 1946-1954.

14. Nielsen, T. Environ. Sci. Technol. 1984, 18, 157-163.

15. Nielsen, T.; Seitz, B.; Ramdahd, T. Atmos. Environ. 1984, 18, 2159-2165.

16. Pitts, J. N., Jr.; Arey Sweetman, J.; Zielinska, B.; Winer, A. M.; Atkinson, R. Atmos. Environ. 1985, 19, 1601-1608.

17. Arey, J.; Zielinska, B.; Atkinson, R.; Winer, A. M.; Ramdahl, T.; Pitts, J. N., Jr. Atmos. Environ. 1986, 20, 2339-2345.

18. Ramdahl, T.; Zielinska, B.; Arey, J.; Atkinson, R.; Winer, A. M.; Pitts, J. N., Jr. Nature 1986, 321, 425-427.

19. Arey, J.; Zielinska, B.; Atkinson, R.; Winer, A. M. Atmos. Environ. 1987, 21, 1437-1444.

20. Zielinska, B.; Arey, J.; Atkinson, R. In Nitroarenes; Howard, P. C., Ed,; Plenum Press: New York, 1990; pp 73-84.

21. Kamens, R. M.; Fan, Z.-H.; Yao, Y.; Chen, D.; Chen, S.; Vartiainen, M. Chemosphere 1994, 28, 1623-1632.

22. Arey Sweetman, J.; Zielinska, B.; Atkinson, R.; Ramdahl, T.; Winer, A. M.; Pitts, J. N., Jr. Atmos. Environ. 1986, 20, 235-238.

23. Atkinson, R.; Winer, A. M.; Pitts, J. N., Jr. Atmos. Environ. 1986, 20, 331-339.

24. Zielinska, B.; Arey, J.; Atkinson, R.; Ramdahl, T.; Winer, A. M.; Pitts, J. N., Jr. J. Am. Chem. Soc. 1986, 108, 4126-4132.

25. Brorström, E.; Grennfelt, P.; Lidskog, A. Atmos. Environ. 1983, 17, 601-605.

26. Zielinska, B.; Arey, J.; Ramdahl, T.; Atkinson, R.; Winer, A. M. J. Chromatogr. 1986, 363, 382-386.

27. Lindskog, A.; Brorström-Lunden, E.; Alfheim, I.; Hagen, I. Sci. Total Environ. 1987, 61, 51-57. 
28. Arey, J.; Zielinska, B.; Atkinson, R.; Winer, A. M. Environ. Sci. Technol. 1988, 22, 457-462.

29. Schuetzle, D.; Lewtas, J. Anal. Chem. 1986, 58, 1060A-1075A.

30. Levsen, K. Fresenius Z. Anal. Chem. 1988, 331, 467-478.

31. Robbat, A., Jr.; Corso, N. P.; Doherty, P. J.; Wolf, M. H. Anal. Chem. 1986, 58, 2078-2084.

32. Yu, W. C.; Fine, D. H.; Chiu, K. S.; Biemann, K. Anal. Chem. 1984, 56, 1158-1162.

33. Tomkins, B. A.; Brazell, R. S.; Roth, M. E.; Ostrum, V. H. Anal. Chem. 1984, 56, 781-786.

34. Niles, R.; Tan, Y. L. Anal. Chim. Acta 1989, 221, 53-63.

35. Paschke, T.; Hawthorne, S. B.; Miller, D. J.; Wenclawiak, B. J. Chromatogr. 1992, 609, 333-340.

36. Hayakawa, K.; Butoh, M.; Miyazaki, M. Anal. Chim. Acta 1992, 266, 251-256.

37. Jin, Z.; Rappaport, S. M. Anal. Chem. 1983, 55, 1778-1781.

38. Schilhabel, J.; Levsen, K. Fresenius Z. Anal. Chem. 1989, 333, 800-805.

39. Henderson, T. R.; Sun, J. D.; Royer, R. E.; Clarck, C. R.; Li, A. P.; Harvey, T. M.; Hunt, D. H.; Fulford, J. E.; Lovette, A. M.; Davidson, W. R. Environ. Sci. Technol. 1983, 17, 443449.

40. Tejada, S. B.; Zweidinger, R. B.; Sigsby, J. E., Jr. Anal. Chem. 1986, 58, 1827-1834.

41. Veigl, E.; Posch, W.; Lindner, W.; Tritthart, P. Chromatographia 1994, 38, 199-206.

42. MacCrehan, W. A.; May, W. E.; Yang, S. D.; Benner, B. A., Jr. Anal. Chem. 1988, 60, 194-199.

43. Galceran, M. T.; Moyano, E. Talanta 1993, 40, 615-621.

44. Spitzer, T. J. Chromatogr. 1993, 643, 43-49.
45. Kamiura, T.; Kawaraya, T.; Tanaka, M.; Nakadoi, T. Anal. Chim. Acta 1991, 254, 27-31.

46. Oehme, M.; Mane, S.; Stray, H. J. High Res. Chromatogr. 1982, 5, 417-423.

47. Nielsen, T. Anal. Chem. 1983, 55, 286-290.

48. Stray, H.; Manø, S.; Mikalsen, A.; Oehme, M. J. High Res. Chromatogr. 1984, 7, 74-82.

49. Ciccioli, P.; Cecinato, A.; Brancaleoni, E.; Liberti, A. /. High Res. Chromatogr. 1988, 11, 306-312.

50. Matsushita, H.; Iida, Y. I. High Res. Chromatogr. 1986, 9, 708-711.

51. Hayakawa, K.; Murahashi, T.; Butoh, M.; Miyazaki, M. Environ. Sci. Technol. 1995, 29, 928-932.

52. Arey, J.; Zielinska, B. J. High Res. Chromatogr. 1989, 12, 101-105.

53. Korfmacher, W. A.; Rushing, L. G.; Arey, J.; Zielinska, B.; Pitts, J. N., Jr. J. High Res. Chromatogr. 1987, 10, 641-646.

54. Lothrop, G.; Hefner, E.; Alfheim, I.; Moller, M. Science 1980, 209, 1037-1039.

55. Ramdahl, T.; Urdal, K. Anal. Chem. 1982, 54, 2256-2260.

56. Sigvardson, K. W.; Birks, J. W. J. Chromatogr. 1984, 316, 507-518.

57. LaCourse, D. L.; Jensen, T. E. Anal. Chem. 1986, 58, 1894-1895.

58. Rappaport, S. M.; Jin, Z. L.; Xu, X. B. J. Chromatogr: 1982, 240. 145-154.

59. Liberti, A.; Ciccioli, P.; Cecinato, A.; Brancaleoni, E.; Di Palo, C. J. High Res. Chromatogr. 1984, 7, 389-397.

60. Heinis, T.; Chowdhury, S.; Kebarle, P. Org. Mass Spectrom. 1993, 28, 358-365. 